Conf- $-950 / 51--5$

UCRL-JC-119213

PREPRINT

\title{
Signatures of Testing: On-Site. Inspection Technologies
}

\author{
J.J. Zucca, C. Carrigan, P. Goldstein, \\ S.P. Jarpe, J. Sweeney, W.L. Pickles \\ Lawrence Livermore National Laboratory \\ B. Wright \\ Los Alamos National Laboratory \\ This was prepared for submittal to the \\ North Atlantic Treaty Organization \\ Advanced Study Institute Meeting \\ Alvor, Algarve, Portugal \\ January 23-February 2, 1995
}

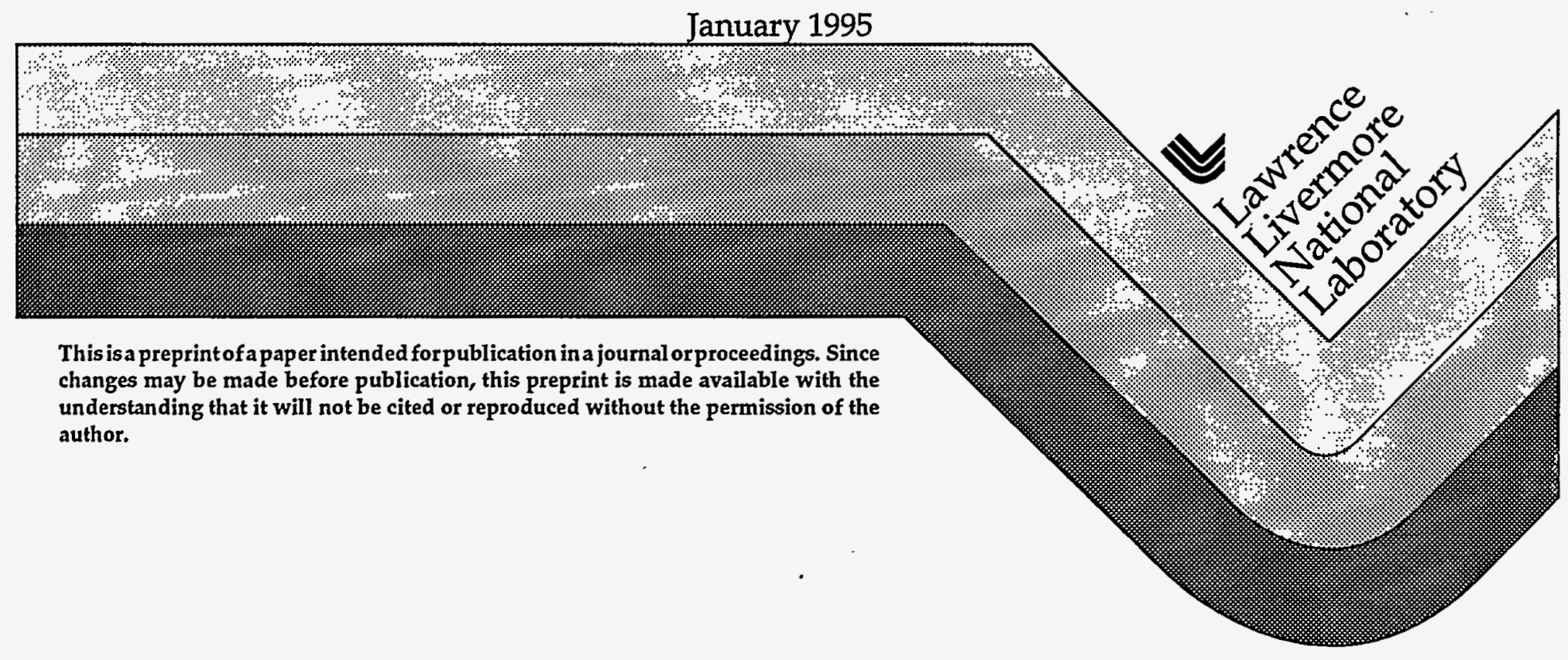




\section{DISCLAIMER}

This document was prepared as an account of work sponsored by an agency of the United States Government. Neither the United States Government nor the University of California nor any of their employees, makes any warranty, express or implied, or assumes any legal liability or responsibility for the accuracy, completeness, or usefulness of any information, apparatus, product, or process disclosed, or represents that its use would not infringe privately owned rights. Reference herein to any specific commercial product, process, or service by trade name, trademark, manufacturer, or otherwise, does not necessarily constitute or imply its endorsement, recommendation, or favoring by the United States Government or the University of California. The views and opinions of authors expressed herein do not necessarily state or reflect those of the United States Government or the University of California, and shall not be used for advertising or product endorsement purposes. 


\title{
SIGNATURES OF TESTING: ON-SITE INSPECTION TECHNOLOGIES
}

\author{
J. J. Zucca, ${ }^{1}$ C. Carrigan, ${ }^{1}$ P. Goldstein, ${ }^{1}$ S. Jarpe, ${ }^{1}$ \\ J. Sweeney, ${ }^{1}$ W. L. Pickles, ${ }^{1}$ and B. Wright ${ }^{2}$ \\ ${ }^{1}$ Lawrence Livermore National Laboratory \\ P. O. Box 808, Livermore, CA, 94551, USA \\ ${ }^{2}$ Los Alamos National Laboratory \\ P. O. Box 1663, Los Alamos, NM, 87545, USA
}

\section{Introduction}

This paper describes the phenomenology of nuclear explosions and technologies for their detection as relevant to On-Site Inspection (OSI) for a comprehensive test-ban (CTB). Our experience with the U.S. nuclear test program which has been primarily carried out at the Nevada Test Site (NTS) and in the Pacific Ocean [1].

The goals of OSI are to resolve ambiguous events, reduce uncertainty, deter attempts at evasion, and provide responsive and technically competent means of confirming the occurrence of a nuclear explosion should deterrence fail. These goals would include finding evidence of an evasive nuclear explosion or evidence that the event was non-nuclear, such as an earthquake or large chemical explosion.

\section{Underground Nuclear Explosion Phenomenology}

At NTS, the majority of nuclear test explosions have been emplaced underground using a vertical bore hole and standard containment practices. Under these conditions, the ground surface above the explosion may collapse within a few hours after detonation, producing a surface crater. Furthermore, there are numerous signs of activity such as new roads and drill pads. While these features do not necessarily indicate a nuclear explosion, they are suggestive evidence that could focus the efforts of an OSI team.

Alternatively, nuclear test explosions may be emplaced in a tunnel, as is the normal practice in Rainier Mesa, or in other underground workings at NTS. In addition, many explosions in Rainier Mesa are "overburied;" that is, they do not collapse to form a crater. However, the surface ground zero will most likely still undergo spallation. The ground surface above a horizontally emplaced explosion may not show any visual signs of emplacement activity because no 


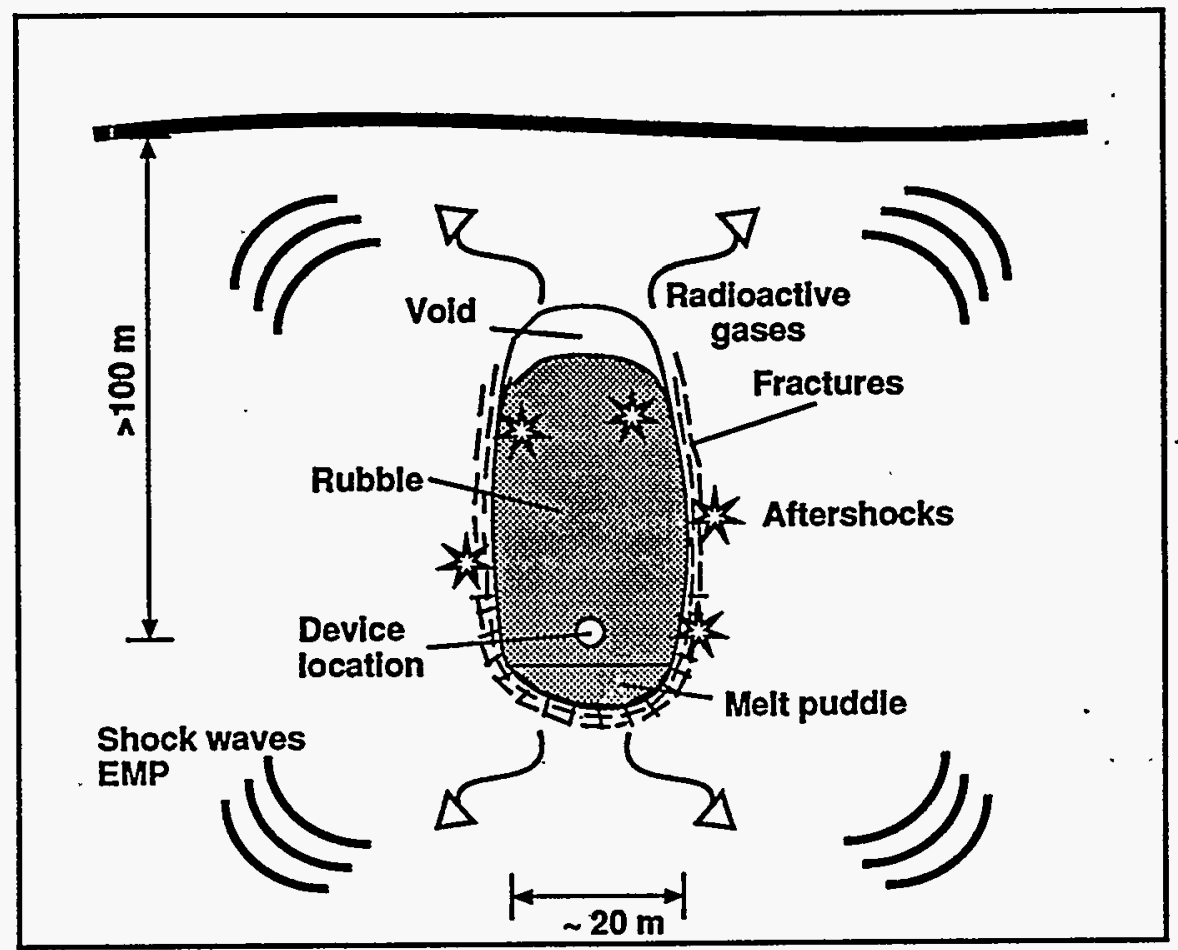

Figure 1. Cross section of underground explosion phenomenology relevant to OSI for an explosion of a few kilotons.

drilling or other activity at surface ground zero is necessary. Figure 1 shows a representative cross section of an overburied explosion with phenomena that are important for OSI.

On-site nuclear explosion phenomena, when observed, may be divided into two categories: (1) direct evidence, i.e., phenomena that provide incontrovertible proof of the occurrence of a nuclear explosion, and (2) indirect evidence, i.e., phenomena that may be indicative of the occurrence of a nuclear explosion and can be used to focus the search for direct evidence. However, indirect evidence could be the result of other activity. On-site nuclear explosion phenomena may also be classified according to how long after the explosion they can be observed. For the purposes of underground explosions, we define three classes: (1) short-lived, up to 1 month duration, (2) medium-lived, up to 2 years in duration, and (3) long-lived, up to 5 years in duration (and beyond in some cases). 


\subsection{SHORT LIVED ( $<1$ MONTH) PHENOMENA}

\subsubsection{Aftershocks}

Aftershocks are produced from most well-coupled underground seismic events. They can be used to focus the search pattern in the case of an explosion, and they may be the only evidence to indicate the event was an earthquake. The aftershock production rate falls off with time. The number of aftershocks produced after a well-coupled test is primarily dependent on the local geologic conditions; explosions in hard rock tend to produce 50 times as many aftershocks as do explosions in soft rock. At the other extreme, explosions in salt produce few if any aftershocks. While there are no data on aftershock production for decoupled events, theoretically a completely decoupled event would produce few if any aftershocks. As the decoupling becomes less effective, the potential for aftershock generation should increase until it approaches the levels measured for well-coupled events.

Aftershock production is also dependent on yield, but only weakly-kiloton explosions appear to produce about as many aftershocks as explosions with yields in the tens of kilotons (Figure 2). Explosion aftershocks tend to cluster around the detonation point in a localized distribution but may also be distributed along local fault planes [2]. Earthquakes of equivalent magnitude and in equivalent geologic conditions tend to generate 10 times as many aftershocks as explosions. Earthquake aftershocks also can occur much deeper than explosion aftershocks and tend to be distributed along planes. Recording 10 to 20 aftershocks may be necessary to distinguish planar from clustered distributions depending on local geologic conditions. Aftershocks from nuclear explosions of a few kilotons tend to be quite small, between magnitude 1 and -3 (Figure 2). In NTS geologic conditions, they can be detected only by a local seismograph station within a few kilometers of the epicenter.

\subsubsection{Xenon Gas}

Xenon gas is a primary product of a nuclear explosion. Two isotopes are of interest for OSI, xenon-135 and xenon-133, which have half-lives of $9 \mathrm{hr}$ and 5 days, respectively. The probability of detection is dependent upon the transport time of the gases to the surface. If arrival at the surface occurs within two weeks, detection probability is good. If transport is delayed beyond a month after the event, detection probability is very low. Transport time could vary depending on the depth and method of burial, weather, and geologic conditions. Rapid arrival at the surface would most likely be the result of dynamic venting, which could release other radioactive products as well. 

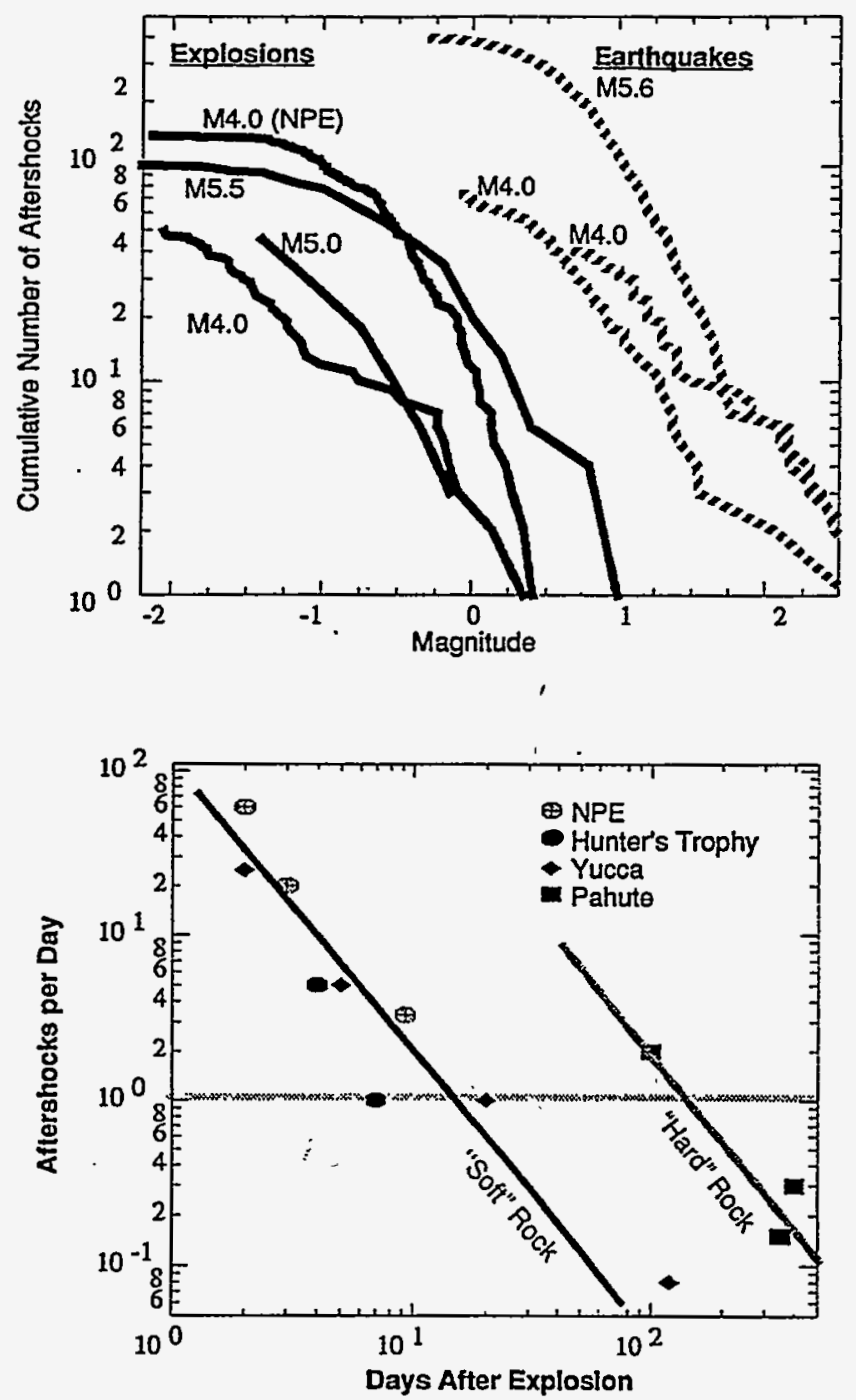

Figure 2. Explosion aftershock data for NTS events. a) Comparison of cumulative number and size for some aftershock sequences. $M=$ magnitude of the main event. b) Aftershock production rate for some events. NPE = non-proliferation event. 


\subsection{MEDIUM LIVED ( $<2$ YR.) PHENOMENA}

\subsubsection{Surface Changes From Ground Spallation}

The ground surface above a nuclear explosion is often thrown into the air a meter or more when the shock wave from the explosion interacts with the ground surface. This violent shaking may cause local rockfall and surface cracking, alter the reflectance and thermal properties of the ground surface, and stress plants. Reflectance properties of the ground may also be altered by wind and rain. In the absence of extensive wind and rain, explosion-induced ground and plant changes would be observable for up to a month or two.

\subsubsection{Argon and Other Radioactive Gasses}

Argon-37 gas, with half-life of 35 days, is a secondary product of a nuclear explosion. It is formed when the intense radiation from the explosion interacts with calcium in the surrounding geologic material and within the device itself. Because of its relatively short half-life, its worldwide background is quite low. Detection of argon-37 is very strong evidence of the recent occurrence of a nuclear test. Other gases are also present such as krypton-85 (11-year half-life), but have a relatively large global background. Detection of these gases does not constitute incontrovertible evidence of a nuclear explosion.

As with xenon, argon and other gases must be transported to the surface in order to be detected by an inspection team, but their longer half-lives allow them to be detectable much later compared to xenon-up to approximately two years in the case of argon. Therefore, transport to the surface can be by the much slower seepage process that could take many months for a well-contained explosion. To optimize detection, gas samples should be taken only during atmospheric lows because gas flows into the ground during periods of highs.

\subsubsection{Changes in Ground Water Level}

After an underground explosion, the ground water is pushed away from the detonation point and then subsequently flows back. Changes in water table level may be observed up to several hundred meters from the detonation point. Depending on local conditions, the ground water level may require a month or more to return to normal. Thus, ground water levels could be monitored for a period of weeks; however, normal changes in ground water level due to seasonal and other changes may be difficult to distinguish from those caused by an explosion.

\subsubsection{Human Generated Artifacts}

Underground nuclear explosions require extensive preparations. A hole or tunnel must be excavated and cables installed to detonate the explosion and record diagnostic information. Artifacts of the activity include roads, underground metal pipe, and spoil piles (i.e., tailings). None or all of these may be present depending on whether the explosion was emplaced in a drill hole or tunnel and the degree of clean-up after the explosion. At NTS, these artifacts 
are visible many years after the explosion, but an evader would attempt to conceal such evidence. A rough guess is that these features, if they exist, might be visible for up to several months.

\subsection{LONG LIVED ( $>2$ YR.) PHENOMENA}

\subsubsection{Surface Cratering}

Under normal NTS practices, the ground above an explosion may collapse to form a crater within a few hours. Once formed, the crater could persist for hundreds to thousands of years. Although they are strong evidence of a nuclear explosion, collapse craters may also be associated with volcanic or mining activity. It is possible to avoid collapse by deeply burying the device.

\subsubsection{Underground Cavity And Rubble Zone}

During an underground nuclear explosion, the surrounding geologic material is vaporized and crushed, which creates an underground cavity. Depending on the emplacement depth and the geologic conditions, the roof of the cavity could start to collapse and create a rubble column that rises toward the surface. If the column reaches the surface, it will create a surface crater. The cavity and rubble zone is a very long-lived feature and could persist for geologic periods of time.

\subsubsection{Residual Underground Radioactivity}

As the detonation point begins to cool after an underground explosion, the vapor condenses and eventually forms a glassy material on the floor of the cavity. This material is highly radioactive and contains many long-lived fission. products. A sample from this region would be recognizable as a nuclear explosion product for tens to hundreds of years.

\section{Underground Explosion Detection Technologies}

OSI technologies may be divided into two categories: (1) technologies that can be used to narrow the search pattern and pinpoint the detonation point of the explosion or provide evidence that the event was an earthquake, and (2) technologies that can be used to retrieve a sample of primary or secondary explosion products.

\subsection{TECHNOLOGIES FOR NARROWING SEARCH PATTERN}

\subsubsection{Overhead Visual Inspection}

This technology would be used to look for human-generated artifacts and explosion craters. Overhead inspection would consist of an aircraft overflight of the inspection area. It would be used to update maps or satellite images and choose sites for more detailed inspections. The inspector could be limited to 
visual observation, positioning equipment, and visible light photography and/or video. The aerial inspection would take less than one day to complete.

\subsubsection{On-Ground Visual Inspection}

On the ground, inspectors could walk specific regions to look for traces of equipment or other artifacts that indicate testing activity. Observations of ground cracking and rock fall could show whether (and where) an underground explosion had taken place and aid in the siting of sampling equipment. An onground visual inspection would require at least a week.

\subsubsection{Overhead Multispectral Imaging}

This technology would be used to detect spalled ground. Multispectral imaging could be performed using imagery from satellites and/or low-flying aircraft. Such imaging produces large amounts of data which, because of the time required for data reduction and interpretation, may limit the usefulness of this technology for narrowing the search area. Plant stress analysis may be possible only with extremely high resolution imagery $(<1 \mathrm{~m})$. Such resolution is possible only with low-altitude overflights and may be considered intrusive. Multispectral imaging data could be collected completely with two overflights; one during the day and one at night.

\subsubsection{Local Seismic Monitoring}

This technology would be used to detect and locate aftershocks and other local seismic events. Equipment would consist of portable short-period seismographs. Global Positioning System equipment is needed to locate the stations and provide accurate timing. Fifteen or more stations would be required for a deployment. Minimum data collection time in the field is one week. For a local seismograph network of 15 stations to be effective, the inspection area needs to be 100 square kilometers or less. If the initial inspection area is larger, initial narrowing will need to be accomplished by some other method, such as aerial inspection or additional seismographs. A van-sized field analysis center is required to provide on-site preliminary analysis so that the search may be managed in the field.

\subsubsection{Magnetic Field Mapping}

This technology would be used to detect buried metal objects such as wires and pipes, i.e., human-generated artifacts. Magnetometers range in size from handheld varieties for mapping localized regions to airborne probes for large regions. Localized on-ground mapping would require a week or more; airborne mapping could be completed in a day.

\subsubsection{Gravity Field Mapping}

This technology detects differential mass distribution underground. It has the potential to be practical for detecting large decoupling cavities in relatively small areas $\left(\sim 10 \mathrm{~km}^{2}\right)$, depending on the size of the cavity, local topography, 
and geology. It is not practical to detect explosion-generated cavities or welltamped explosions since mass is not missing in these cases; instead, it is redistributed. The gravity signature in such a case is likely to be small and difficult to distinguish from the background field. Gravity field mapping requires precision location and elevation surveying down to millimeter accuracy (currently unavailable on GPS) in addition to detailed maps of the surrounding region. Its usefulness could be confounded by the presence of nearby legitimate mine workings. A gravity survey would require two to three weeks to cover a localized region ( $-10 \mathrm{sq} . \mathrm{km})$ where a mined cavity is already suspected to exist.

\subsubsection{Geophysical Sounding}

This technology would be used to search for explosion generated cavities and rubble zones, decoupling cavities, and possibly changes in ground water level. It would consist of equipment for electromagnetic sounding, seismic reflection and refraction, direct-current resistivity, and other similar techniques. These systems are labor intensive and can involve deployment. of many sensors together with their associated cables and power sources. Such surveys cover only localized regions and require 10 to 20 people several weeks on-site depending on the level of detail required and the local conditions. A key issue with geophysical sounding is to avoid confusing the desired target with other features. This can be quite challenging in areas of complicated geologic structure or mining operations.

\subsection{TECHNOLOGIES FOR RETRIEVING A RADIOACTIVE SAMPLE}

\subsubsection{Gas Sampling}

This technology would be used to detect xenon and argon gasses. Gas samples can be taken from under tarps or probes driven into the ground. Atmospheric pumping can be a significant component of gas transport, therefore gas samples should be taken at times of atmospheric lows [3]. Analysis capability should exist in the field so that search parameters could be changed during the inspection. Available technology would fit into a van-type vehicle.

\subsubsection{Drilling}

This technology would be used to look for underground residual radioactivity which would be present in the glassy material on the floor of the cavity and in the rubble zone. This zone is likely to present a drilling target tens of meters wide. Directional drilling and blowout protection has been used for many years by NTS and the oil industry. The U.S. used a light-weight drill rig at the Semipalatinsk Test Site in the Former Soviet Union for the Joint Verification Experiment. Costs to transport such equipment for routine OSIs are likely to be prohibitive. However, in many instances, costs could be significantly reduced by using local drilling contractors. 


\section{Open-Water Explosions Phenomenology}

In contrast to the hundreds of tests that have been conducted underground, the U.S. has conducted only a handful of open-water nuclear tests. Nuclear explosion effects at sea are controlled by the distance between the detonation point and the surface of the water. Although a myriad of effects can be achieved by varying the depth, two sets of initial conditions cover most the phenomena important for OSI: (1) deep underwater explosions and (2) explosions near the surface, either above or below. At the time of detonation of a deep explosion, the surrounding water ( $>200 \mathrm{~m}$ for 1 kiloton) is heated and part of it is vaporized. The resulting bubble, which is composed primarily of steam, immediately starts to rise to the surface. Because of the radial momentum of the gas in the bubble and the pressure of the surrounding water, it begins to expand and contract. These oscillations give rise to the "bubble pulse" which is observed in the hydroacoustic signals. The bubble and its contents condense as they rise. For deep explosions, the bubble may not break the surface.

Deep explosions deposit most of the explosion products into the water, except for the non-condensable gaseous components such as xenon and krypton. Energy from the explosion raises the water temperature and causes upwelling of explosion products to a location above the thermocline. This creates a pool or slurry of explosion debris within a few hundred meters of the surface. For a 1-kt explosion, the slurry is a kilometer or two in diameter after $24 \mathrm{hr}$ and expands to approximately $10 \mathrm{~km}$ after a week. At the same time, ocean currents and surface conditions begin to affect the slurry. Ocean currents can be as high as 4 $\mathrm{km} / \mathrm{hr}$. If we assume an average current rate of $2 \mathrm{~km} / \mathrm{hr}$, in $12 \mathrm{hr}$ the pool could move just outside the nominal $1000 \mathrm{~km}^{2}$ error ellipse from the remote monitoring system. In general, the slurry should be detectable from a lowiflying aircraft for a week or two and from the ocean surface, either from a ship or air-dropped buoy, for up to a month or two. Figure 3 summarizes the phenomena for a deep explosion and gives an approximate timeline. The figure shows a spray dome at the surface, which forms if the bubble reaches the water surface still intact.

Explosions near the surface, either above or below, are distinguished from deep explosions in that a bubble pulse does not form, significant heating of the water does not occur, and more explosion products are injected into the atmosphere. The radioactive cloud from a shallow explosion contains particulates as well as non-condensable gasses and tends to be restricted to the lower atmosphere where is it susceptible to removal by rain and snow. Depending on weather conditions, the cloud will be dispersed within a week or two. If the explosion occurs during heavy rain, virtually all of the radioactivity that is injected into atmosphere can be rained out in a few hours and within several hundred kilometers of the detonation point-except for the noncondensable components. 

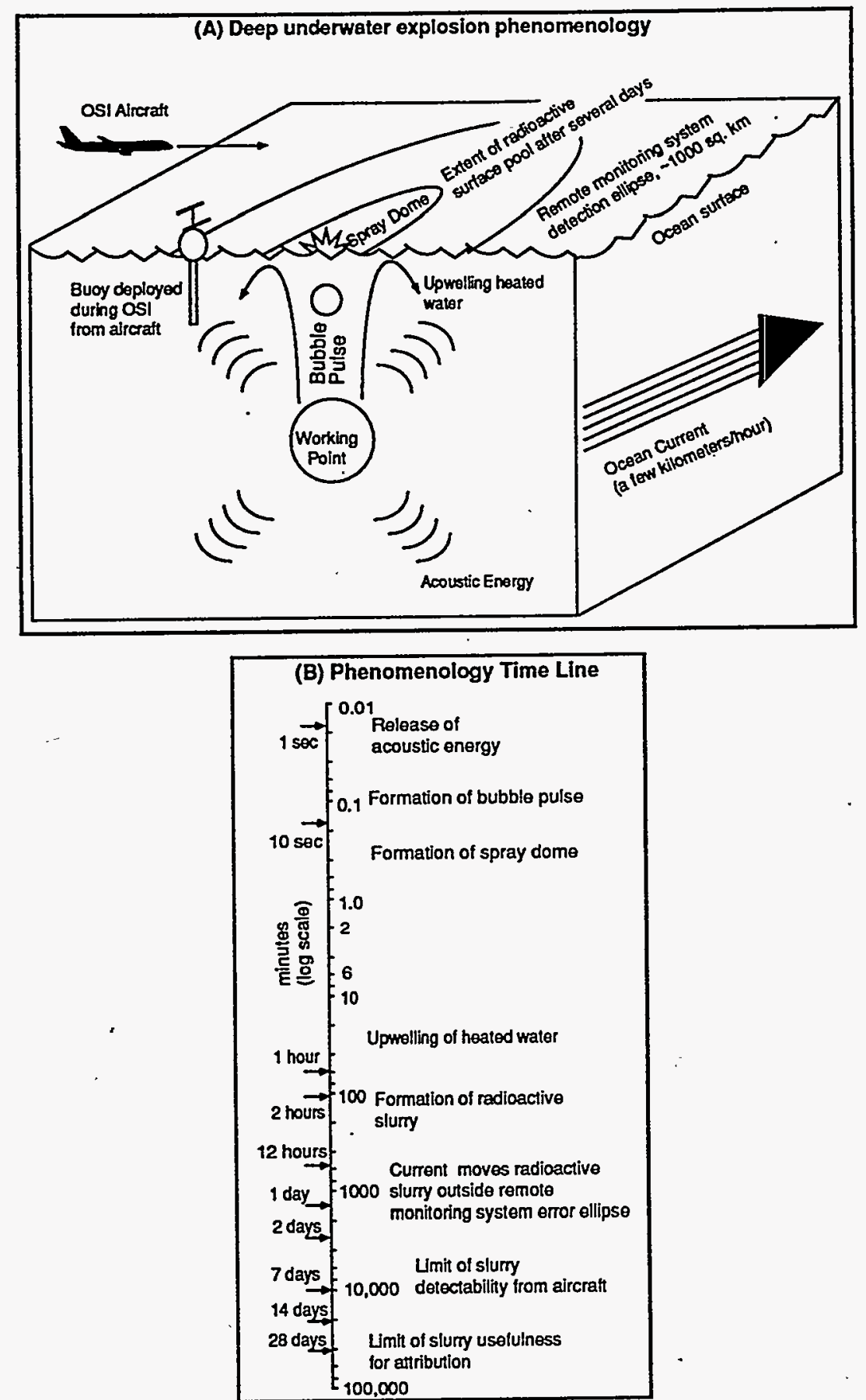

Figure 3. Underwater explosion phenomenology. (A) Schematic diagram of a deep underwater explosion. (B) Timeline of underwater explosion phenomenology. 


\section{Open-Water OSI Technologies}

To respond within several days of the event, an aircraft would need to be dedicated to the mission. Once on station, the inspectors will need to take water and air samples to attempt to locate the radioactive slurry and cloud. If the inspectors arrive at the site more than 12 hours after the event, weather and water current conditions must be known so that the "cloud" trajectories away from the location given by the remote monitoring system can be estimated. In the general case, the prevailing winds and water currents will be in different directions, causing the slurry and cloud to drift away from the detonation site in different directions.

Gas and aerosol samples can be taken with an aircraft using technology being developed for the permanent radionuclide monitoring network, but will require some re-engineering. Assuming that the aircraft can stay on station only several hours, it is unlikely that detailed analysis of the gas samples can be made on the aircraft since several hours are required to separate the gases and count the decay products. However, gamma spectra can be taken immediately of aerosol samples and can be used to direct the search in real time. A towed or wing-mounted gamma sensor could be used to search for high concentrations of particulates in the air or on the ocean surface. The results from such an instrument would be available in real time and could also be used to help direct the search.

Water samples could be taken directly using a sampler deployed on a line from the aircraft. After the slurry has been located, greater sensitivities could be obtained through deploying a buoy that could pump a volume of water through a filter and later recover the filtered products for analysis. A gamma spectrum sample could be taken in the aircraft, but detailed analysis would need to be done after the search. Plankton will concentrate some of the fission products and may also be collected and analyzed several months later. Once the slurry is located from the air, a ship could be sent to collect water samples.

\section{Zerotime OSI}

The discussion so far has assumed that the inspection is occurring after the event. Inspectors could also be invited in before a large legitimate explosion to monitor for the occurrence of a nuclear explosion in the vicinity. In addition to monitoring for the above discussed phenomena, one could also monitor for prompt phenomena-in particular electromagnetic pulse and the hydrodynamic shock wave.

During an underground nuclear explosion, a broad band electromagnetic pulse is created by virtue of a rapid charge separation and recombination [1]. Unless there are wires or other waveguides for this energy to reach the surface, frequencies greater than a few hundred Hertz will be strongly attenuated by the overburden. However, a low frequency $(\sim 10 \mathrm{~Hz})$ pulse may be observed 
within several kilometers of ground zero. Chemical explosions also generate electromagnetic puises [4]. Limited empirical data from equivalent nuclear and chemical explosions suggests that a reduced field strength and a spectrum shift to lower frequencies may be used to discriminate between chemical and nuclear explosions.

Given that a network of accelerometers are deployed in the region around the declared explosion and the surrounding vicinity, the zerotime recordings should contain the superposition of the evasive and declared explosions' signatures. Array processing techniques could be used to separate the signals and estimate a location.

This work was performed under the auspices of the U.S. Department of Energy by Lawrence Livermore National Laboratory under contract No. W-7405-Eng-48. 


\section{References}

1. Glasstone, S., and Dolan. O. J. (1977) The eEfects of Nuclear Weapons, U.S. Dept. of Defense and the Energy Res. and Dev. Administration, Third edition:

2. Edwards, C. L., M. D. Harper, T. A. Weaver, D. J. Cash, J. M. Ray, and E. F. Homuth (1983) Microcarthquake Activity Associated with Underground Nuclear Testing at the Nevado Test Site, Los Alamos National Laboratory, Los Alamos, NM, LA-8552-MS.

3. Nilson, R. H., Peterson, E. W., Lie, K. H., Burkhard, N. R., and Hearst, J. R. (1991) Atmospheric pumping: A mechanism causing vertical transport of contaminated gases through fractured permeable media, J. Geophys. Res. 96, 21933-21948.

4. Kelly, B. (1993) EMP from Chemical Explosions, Los Alamos National Laboratory, Los Alamos, NM, LA-UR-93-983. 